\title{
Black Holes or Dark Stars-What Follows from the General Relativity Theory
}

\author{
Valery V. Vasiliev \\ Institute of Problems in Mechanics, Russian Academy of Sciences, Moscow, Russia \\ Email: vvvas@dol.ru
}

How to cite this paper: Vasiliev, V.V. (2017) Black Holes or Dark Stars-What Follows from the General Relativity Theory. Journal of Modern Physics, 8, 1087-1100. https://doi.org/10.4236/jmp.2017.87070

Received: May 11, 2017

Accepted: June 13, 2017

Published: June 16, 2017

Copyright $\odot 2017$ by author and Scientific Research Publishing Inc. This work is licensed under the Creative Commons Attribution International License (CC BY 4.0).

http://creativecommons.org/licenses/by/4.0/

\begin{abstract}
The paper is concerned with spherically symmetric static problem of the Classical Gravitation Theory (CGT) and the General Relativity Theory (GRT). First, the Dark Stars, i.e. the objects that are invisible because of high gravitation preventing the propagation of light discovered in the 18th century by $\mathrm{J}$. Michel and P. Laplace are discussed. Second, the Schwarzchild solution which was obtained in the beginning of the 20th century for the internal and external spaces of the perfect fluid sphere is analyzed. This solution results in singular metric coefficients and provides the basis of the Black Holes. Third, the general metric form in spherical coordinates is introduced and the solution of GRT problem is obtained under the assumption that gravitation does not affect the sphere mass. The critical sphere radius similar to the Black Hole horizon of events is found. In contrast to the Schwarzchild solution, the radial metric coefficient for the sphere with the critical radius referred to as the Dark Star is not singular. For the sphere with radius which is less than the critical value, the GRT solution becomes imaginary. The problem is discussed within the framework of the phenomenological theory which does not take into account the actual microstructure of the gravitating objects and, though the term "star" is used, the analysis is concerned with a model fluid sphere rather than with a real astrophysical object.
\end{abstract}

\section{Keywords}

General Relativity, Spherically Symmetric Problem, Black Holes, Dark Stars

\section{Introduction}

The existence of Dark Stars was predicted by J. Michel in 1783 and P. Laplace in 1796 [1] [2]. As known, the escape velocity for a spherical body with radius $R$ and mass $m$ is

$$
v_{e}=c \sqrt{r_{g} / R}
$$


in which $c$ is the velocity of light and

$$
r_{g}=2 m G / c^{2}
$$

is the so-called gravitational radius which depends on the gravitational constant $G$ and the sphere mass. For the sphere with constant density $\mu$ in the Euclidean space

$$
m=4 / 3 \pi \mu R^{3}
$$

Substituting Equations (2) and (3) in Equation (1), we get

$$
v_{e}=2 R \sqrt{2 / 3 \pi \mu G}
$$

According to the reconstructed Laplace calculation, for Earth with density $\mu_{E}$ $=5520 \mathrm{~kg} / \mathrm{m}^{3}$ and radius $R_{E}=6371032 \mathrm{~m}$, Equation (4) yields $V_{e}=11,000 \mathrm{~m} / \mathrm{s}$ which is 27,270 times less than the velocity of light. Because $v_{e}$ in Equation (4) is proportional to $R$ increasing $R$ up to $1.62 \times 10^{11} \mathrm{~m}$ which is 249.6 times higher than the radius of Sun $\left(6.96 \times 10^{8} \mathrm{~m}\right)$ we arrive at $v_{e}=c$. This calculation allowed Laplace to conclude that the star with the density of Earth and the radius which is about 250 times larger than the radius of Sun is not visible and can be referred to as the Dark Star. Later, the idea of Dark Stars based on CGT and the corpuscular model of light was abandoned by Laplace and the subsequent authors [1] [2]. The present paper contains an attempt to revive this idea within the framework of GRT. Under some assumptions, we obtain the solution of GRT equations which corresponds to the object that can be treated as the Dark Star.

\section{General Relativity Equations}

The general form of the line element in spherical coordinates $r, \theta, \varphi$ can be presented as

$$
\mathrm{d} s^{2}=g_{11} \mathrm{~d} r^{2}+g_{22}\left(\mathrm{~d} \theta^{2}+\sin ^{2} \theta \mathrm{d} \varphi^{2}\right)-g_{44} c^{2} \mathrm{~d} t^{2}
$$

For spherically symmetric static problems, the components of the metric tensor $g_{i j}$ depend only on the radial coordinate $r$. Material properties of space are determined by three components of the energy tensor $T_{1}^{1}, T_{2}^{2}=T_{3}^{3}, T_{4}^{4}$ which satisfy the conservation equation [3]

$$
\left(T_{1}^{1}\right)^{\prime}-\frac{g_{22}^{\prime}}{g_{22}}\left(T_{2}^{2}-T_{1}^{1}\right)+\frac{g_{44}^{\prime}}{2 g_{44}}\left(T_{1}^{1}-T_{4}^{4}\right)=0
$$

where $(\ldots)^{\prime}=\mathrm{d}(\ldots) / \mathrm{d} r$.

In accordance with the basic mathematical idea of GRT, Equation (6) is satisfied identically if the tensor $T_{i}^{j}$ is expressed in terms of the metric coefficients by the following Einstein equations:

$$
\begin{gathered}
\chi T_{1}^{1}=\frac{1}{g_{22}}\left[1-\frac{g_{22}^{\prime}}{2 g_{11}}\left(\frac{g_{22}^{\prime}}{2 g_{22}}+\frac{g_{44}^{\prime}}{g_{44}}\right)\right] \\
\chi T_{2}^{2}=-\frac{1}{g_{11}}\left[\frac{g_{22}^{\prime \prime}}{2 g_{22}}+\frac{g_{44}^{\prime \prime}}{2 g_{44}}-\left(\frac{g_{22}^{\prime}}{2 g_{22}}\right)^{2}-\left(\frac{g_{44}^{\prime}}{2 g_{44}}\right)^{2}+\frac{g_{22}^{\prime}}{4 g_{22}}\left(\frac{g_{44}^{\prime}}{g_{44}}-\frac{g_{11}^{\prime}}{g_{11}}\right)-\frac{g_{11}^{\prime} g_{44}^{\prime}}{4 g_{11} g_{44}}\right]
\end{gathered}
$$




$$
\chi T_{4}^{4}=\frac{1}{g_{22}}-\frac{1}{g_{11}}\left[\frac{g_{22}^{\prime \prime}}{g_{22}}-\left(\frac{g_{22}^{\prime}}{2 g_{22}}\right)^{2}-\frac{g_{11}^{\prime} g_{22}^{\prime}}{2 g_{11} g_{22}}\right]
$$

Here,

$$
\chi=8 \pi G / c^{4}
$$

is the GRT gravitational constant. Because the left-side parts of these equations are linked by Equation (6), only two of three Equations (7)-(9) are mutually independent. Traditionally [3], the simplest combination of these equations, including Equations (7) and (9) is used to determine the metric coefficients $g_{11}$ and $g_{44}$ whereas Equation (8) is satisfied identically for any function $g_{22}(r)$ Possible forms of the solution of two equations (7) and (9) with three unknown functions are discussed by Vasiliev and Fedorov [4].

Because the metric coefficients must be positive, introduce new functions, i.e., take $g_{11}=g^{2}(r), g_{22}=\rho^{2}(r), g_{44}=h^{2}(r)$. Consider the external and the internal spaces for the sphere with radius $R$.

For the external empty space $(r \geq R)$ we have $T_{i}^{j}=0$. Then, Equation (6) is satisfied automatically, whereas Equations (7) and (9) reduce to

$$
\frac{1}{\rho_{e}}-\frac{\rho_{e}^{\prime}}{g_{e}^{2}}\left(\frac{\rho_{e}^{\prime}}{\rho_{e}}+\frac{2 h_{e}^{\prime}}{h_{e}}\right)=0, g_{e}^{2}-\left[2 \rho_{e} \rho_{e}^{\prime \prime}+\left(\rho_{e}^{\prime}\right)^{2}-2 \frac{g_{e}^{\prime}}{g_{e}} \rho_{e} \rho_{e}^{\prime}\right]=0
$$

Index " $e$ " corresponds to the external space. Thus, we have two equations for three functions $g_{e}(r), \rho_{e}(r), h_{e}(r)$ and to solve the problem, we need one more equation.

For the internal space of the solid sphere $(0 \leq r \leq R)$,

$$
T_{1}^{1}=\sigma_{r}, T_{2}^{2}=T_{3}^{3}=\sigma_{\theta}, T_{4}^{4}=\mu c^{2}
$$

where $\sigma_{r}$ and $\sigma_{\theta}$ are the radial and the circumferential stresses acting in a solid sphere. Then, Equations (7) and (9) become

$$
\begin{gathered}
\chi \sigma_{r}=\frac{1}{\rho_{i}^{2}}-\frac{1}{g_{i}^{2}} \frac{\rho_{i}^{\prime}}{\rho_{i}}\left(\frac{\rho_{i}^{\prime}}{\rho_{i}}+\frac{2 h_{i}^{\prime}}{h_{i}}\right) \\
\chi \mu c^{2}=\frac{1}{\rho_{i}^{2}}-\frac{1}{g_{i}^{2}}\left[\left(\frac{\rho_{i}^{\prime}}{\rho_{i}}\right)^{2}+\frac{2 \rho_{i}^{\prime \prime}}{\rho_{i}}-\frac{2 \rho_{i}^{\prime} g_{i}^{\prime}}{\rho_{i} g_{i}}\right]
\end{gathered}
$$

Index " 1 " corresponds to the internal space. The conservation equation, Equation (6), takes the form

$$
\sigma_{r}^{\prime}-\frac{2 \rho_{i}^{\prime}}{\rho_{i}}\left(\sigma_{\theta}-\sigma_{r}\right)+\frac{h_{i}^{\prime}}{h_{i}}\left(\sigma_{r}-\mu c^{2}\right)=0
$$

This is actually the equilibrium equation for a solid sphere [5]. As known, to determine the stresses, the equilibrium equation should be supplemented with constitutive equations which link stresses and strains in a deformable solid and with the compatibility equations for the strains. Such equations do not exist in GRT, and this theory cannot be applied to study gravitation in deformable solids [6]. The only particular case for which the solution can be found corresponds to 
the sphere consisting of perfect fluid. In this case, $\sigma_{r}=\sigma_{\theta}=-p(r)$ where $p$ is the pressure in the fluid. Then, Equation (14) reduces to

$$
p^{\prime}+\frac{h_{i}^{\prime}}{h_{i}}\left(p+\mu c^{2}\right)=0
$$

Thus, for the case of a fluid sphere we have three equations, Equations (12), (13) and (15), which include four unknown functions $g_{i}(r), \rho_{i}(r), h_{i}(r)$ and $p(r)$. Again, to solve the problem we need one more equation.

The solution for the external space must satisfy the asymptotic condition according to which it must degenerate into CGT solution with a distance from the sphere, the solution for the internal space must satisfy the regularity condition at the sphere center, and both solutions must meet the boundary conditions on the sphere surface.

\section{Schwarzchild's Solution and Black Holes}

Both external and internal problems for a fluid sphere were solved by $\mathrm{K}$. Schwarzchiuld in 1916. The equation that forms the complete set with the equations presented above was taken in the form $p(r)=r$.

\subsection{External Space}

Putting $p_{e}=r$ in Equations (11), we arrive at

$$
\frac{h_{e}^{\prime}}{h_{e}}=\frac{1}{2 r}\left(g_{e}^{2}-1\right), \frac{\mathrm{d}}{\mathrm{d} r}\left(r-\frac{r}{g_{e}^{2}}\right)=0
$$

The solutions of these equations are

$$
g_{e}^{2}=\frac{1}{1+C_{1} / r}, h_{e}^{2}=C_{2}\left(1+\frac{C_{1}}{r}\right)
$$

The integration constants $C_{1}$ and $C_{2}$ can be found from the asymptotic conditions according to which for $r \rightarrow \infty$ the obtained solution must reduce to the CGT solution that is expressed in terms of the Newton gravitation potential $\varphi$ [7], i.e., to

$$
g_{0}^{2}=1-\frac{2 \varphi}{c^{2}}, h_{0}^{2}=1+\frac{2 \varphi}{c^{2}}, \varphi=-\frac{G m}{r}
$$

As a result, Equations (16) take the well known form [3]

$$
g_{e}^{2}=\frac{1}{1-r_{g} / r}, h_{e}^{2}=1-\frac{r_{g}}{r}
$$

As follows from the derivation of Equations (18), they include the radius $r_{g}$ which is specified by Equation (2) and corresponds to the classical gravitation theory. Equations (18) show that the Schwarzchild solution determines the metric coefficients of the external space in terms of the sphere gravitational radius which depends on the sphere mass only and does not take into account the internal structure of the sphere, i.e., the structure of the source of gravitation. Such situation is typical for CGR, i.e., for the solution in Equations (17), which is 
unique. However, for GRT the situation can be different, because the function $g_{22}=\rho^{2}(r)$ in Equation (5) can be chosen in different forms. Consider, for example, De-Donder-Fock solution which satisfies the so-called harmonic coordinate condition. Omitting mathematical formulation and physical interpretation of this condition which can be found elsewhere [8] [9], presume that the function $\rho(r)$ has the following form: $\rho(r)=r+r_{g} / 2$. Then, the solutions of Equations (11) become [8] [9]

$$
g_{e}^{2}=\frac{r+r_{g} / 2}{r-r_{g} / 2}, h_{e}^{2}=\frac{r-r_{g} / 2}{r+r_{g} / 2}
$$

For $r \rightarrow \infty$, these solutions asymptotically reduce to Equations (17). Thus, Equations (19) satisfy all GRT equations and asymptotic conditions, but do not coincide with the Schwarzchild solution in Equations (18).

\subsection{Internal space}

For the internal space of the fluid sphere and $\rho(r)=r$, Equations (12) and (13) reduce to

$$
\begin{gathered}
\frac{1}{r g_{i}^{2}}\left(\frac{1}{r}+\frac{3 h_{i}^{\prime}}{h_{i}}\right)-\frac{1}{r^{2}}=\chi p \\
\frac{1}{r^{2}} \frac{\mathrm{d}}{\mathrm{d} r}\left(r-\frac{r}{g_{i}^{2}}\right)=\chi \mu c^{2}
\end{gathered}
$$

In conjunction with Equation (15) for the pressure we have three equations for three unknown functions $g_{i}(r), h_{i}(r)$ and $p(r)$ For the sphere with constant density $\mu$, the solution of Equation (21) which satisfies the regularity condition at the sphere center $r=0$ is [3]

$$
g_{i}^{2}=\frac{1}{1-\left(\chi \mu c^{2} / 3\right) r^{2}}
$$

This solution must satisfy the boundary condition on the sphere surface according to which $g_{i}(R)=g_{e}(R)$. Using Equations (18), we have

$$
\frac{1}{3} \mu c^{2} R^{3}=r_{g}
$$

and finally get [3]

$$
g_{i}^{2}=\frac{1}{1-r_{g} r^{2} / R^{3}}
$$

Consider Equation (20) for $h_{i}$. Substituting Equation (24), we arrive at

$$
\frac{h_{i}^{\prime}}{h_{i}}=\frac{1}{2}\left[\frac{r}{1-r_{g} r^{2} / R^{3}}\left(\frac{1}{r^{2}}+\chi p\right)-\frac{1}{r}\right]
$$

Substituting Equation (25) in Equation (15) and using Equation (10) for $\chi$, we get the following equation for the pressure in the fluid:

$$
p^{\prime}+\frac{r_{g} r}{2\left(R^{3}-r_{g} r^{2}\right)}\left(p+\mu c^{2}\right)\left(1+\frac{3 p}{\mu c^{2}}\right)=0
$$


The general solution of this equation is [3]

$$
p=-\mu c^{2} \frac{\sqrt{1-r_{g} r^{2} / R^{3}}-C_{3}}{\sqrt{1-r_{g} r^{2} / R^{3}}-3 C_{3}}
$$

The integration constant $C_{3}$ can be found from the boundary condition on the sphere surface according to which $p(R)=0$. The resulting expression for the pressure is [3]

$$
p=-\mu c^{2} \frac{\sqrt{1-r_{g} r^{2} / R^{3}}-\sqrt{1-r_{g} / R}}{\sqrt{1-r_{g} r^{2} / R^{3}}-3 \sqrt{1-r_{g} / R}}
$$

Thus, the Schwarzchild solution is specified by Equations (18) for the external space and Equations (24), (25), (28) for the internal space of the fluid sphere with constant density.

\subsection{Analysis}

To demonstrate the specific features of the Schwarzchild solution, consider it for the sphere surface $r=R$. For this surface, we have

$$
\begin{aligned}
& g_{e}=g_{i}=g_{R}=\frac{1}{\sqrt{1-r_{g} / R}}, \\
& h_{e}=h_{i}=h_{R}=\sqrt{1-r_{g} / R}
\end{aligned}
$$

As can be seen, for the sphere with radius $R=r_{g}, g_{R}$ becomes singular and $h_{R}$ is zero. This result has not been accepted by A. Einstein [10] who expected that GRT could not be valid for the sphere with radius close to $r_{g}$. Later, the singularity of the Schwarzchild solution gave rise to the idea of Black Holes and $r_{g}$ was referred to as the radius of the horizon of events of the Black Hole [1] [2]. However, recent results in Mechanics of Solids [11] allow us to conclude that singular solutions do not have physical nature and are the formal consequences of the discrepancy between physical and mathematical models. Particularly, the mathematical model of space specified by Equation (5) is simplified by the condition $\rho(r)=r$ which is not properly justified. As a result, the Schwarzchild solution suffers from several shortcomings discussed below.

First, the critical radius $r_{g}$ in GRT solution in Equations (18) and (24) is the same that in Equation (2) which follows from CGT.

Second, the solution for $g_{i}$ in Equation (24) satisfies the boundary condition on the sphere surface only if Equation (23) is valid. Substituting $r_{g}$ from Equation (2) and $\chi$ from Equation (10) in Equation (23), we can derive the equation for the sphere mass which becomes $m=4 / 3 \pi \mu R^{3}$ and coincides with Equation (3) corresponding to the Euclidean space. However, the space inside the sphere, in accordance with the basic idea of GRT, is Riemannian. The mass of the sphere with constant density corresponding to the metric form for the line element in Equation (5) is

$$
m=4 \pi \mu \int_{0}^{R} g_{i} \rho_{i}^{2} \mathrm{~d} r
$$


Substituting $g_{i}$ from Equation (24) and taking $\rho_{i}=r$, we get

$$
m=\frac{2 \pi}{r_{g}} \mu R^{4}\left(\sqrt{\frac{R}{r_{g}}} \sin ^{-1} \sqrt{\frac{r_{g}}{R}}-\sqrt{1-\frac{r_{g}}{R}}\right) \approx \frac{4}{3} \pi \mu R^{3}\left[1+\frac{3 r_{g}}{10 R}+\frac{9}{56}\left(\frac{r_{g}}{R}\right)^{2}+\cdots\right]
$$

The second part of this equation is the power decomposition with respect to $r_{g} / R$. As follows from Equations (3) and (30), the sphere mass corresponds to the Euclidean space only if $r_{g}=0$. In the general case, $r_{g} \geq 0$ and the sphere mass, corresponding to the Schwarzchild solution must be specified by Equation (30). However, in this case $g_{e}(R) \neq g_{i}(R)$ and the boundary condition for the external and internal spaces cannot be satisfied on the sphere surface.

Third, Equation (28) for the pressure demonstrates rather specific behavior of the function $p(r, R)$ [12]. Consider the pressure at the sphere center. Taking $r=0$ in Equation (28), we get

$$
p_{0}=\mu c^{2} \frac{1-\sqrt{1-r_{g} / R}}{3 \sqrt{1-r_{g} / R}-1}
$$

The denominator of this expression becomes zero if $R=R_{s}=9 / 8 r_{g}=1.125 r_{g}$ [13]. Thus, the pressure becomes infinitely high at the sphere center if $R=R_{s}$. This result is traditionally used to justify the existence of Black Holes [1]. However, $R_{s}$ does not coincide with $r_{g}$. Then, Equation (31) specifies the pressure if $R$ $<R_{s}$ but this pressure is negative (or the density is negative) which does not have physical meaning. Finally, if we use the general solution in Equation (27) and take $r=r_{g}$, we get

$$
p=-\mu c^{2} \frac{\sqrt{1-(r / R)^{2}}-C_{3}}{\sqrt{1-(r / R)^{2}}-3 C_{3}}
$$

On the sphere surface $r=R$, we have $p(R)=-\mu c^{2} / 3$ and the boundary condition $p(R)=0$ cannot be satisfied.

\subsection{Generalized Schwarzchild Solution for the Internal Space}

The original Schwarzchild solution for the internal space discussed in Section 3.2 has been obtained for the sphere with constant density $\mu$. Within the framework of the Schwarzchild assumption, i.e. $\rho(r)=r$, consider the sphere for which $\mu(r)$ is an arbitrary function of the radial coordinate. In this case, the solution of Equation (21) that generalizes the solution in Equation (22) for the constant density is

$$
g_{i}^{2}=\left(1-\chi c^{2} \frac{1}{r} \int_{0}^{r} \mu(r) r^{2} \mathrm{~d} r\right)^{-1}
$$

For the sphere surface, we get

$$
g_{i}^{2}(R)=\left(1-\frac{\chi c^{2}}{4 \pi} m\right)^{-1}
$$

where 


$$
m=4 \pi \int_{0}^{R} \mu(r) r^{2} \mathrm{~d} r
$$

is the sphere mass corresponding to the Euclidean space. If we use Equations (18), (2) and (10) for $g_{e}, r_{g}$ and $\chi$, we can conclude that Equation (32) satisfies the boundary condition $g_{i}(R)=g_{e}(R)$. However actually, the sphere mass with the metric coefficient specified by Equation (32) does not correspond to the Euclidean space.

Thus, in the general case of the sphere whose density is an arbitrary function of the radial coordinate and $\rho(r)=r$, the boundary condition for $g(r)$ can be satisfied only if the geometry of the internal space is Euclidean which is not the case. The reason of this situation follows from Equation (13) which has, in general, the second order. Under the condition $\rho(r)=r$, it reduces to the equation of the first order and the corresponding solution does not contain the proper number of integration constants that are required to satisfy the boundary conditions.

\section{Dark Stars}

\subsection{General Solution}

Consider the general metric form of the line element specified by Equation (5) in which $g_{22}(r)=\rho^{2}(r)$ is some unknown function. For the external space, the second equation in Equations (11) can be reduced to

$$
\frac{1}{\rho_{e}^{\prime}} \frac{\mathrm{d}}{\mathrm{d} r}\left[\frac{\rho_{e}\left(\rho_{e}^{\prime}\right)^{2}}{g_{e}^{2}}\right]=1
$$

and has the following general solution:

$$
g_{e}^{2}=\frac{\rho_{e}\left(\rho_{e}^{\prime}\right)^{2}}{\rho_{e}+C_{4}}
$$

Substituting this result in the first equation in Equations (11) and integrating, we get

$$
h_{e}^{2}=C_{5}\left(1+\frac{C_{4}}{\rho_{e}}\right)
$$

Assume (and further prove) that the following asymptotic conditions are valid for $\rho_{e} \rightarrow \infty$ :

$$
\lim \rho_{e}(r)=r, \lim \rho_{e}^{\prime}(r)=1
$$

i.e., that at a distance from the sphere $\rho_{e}$ degenerates into $r$. Then, Equations (33) and (34) asymptotically reduce to Equations (17) corresponding to CGT if $C_{4}=$ $-r_{g}$ and $C_{5}=1$. Finally,

$$
g_{e}^{2}=\frac{\rho_{e}\left(\rho_{e}^{\prime}\right)^{2}}{\rho_{e}-r_{g}}, h_{e}^{2}=1-\frac{r_{g}}{\rho_{e}}
$$

For the internal space, Equation (13) can be transformed to 


$$
1-\frac{1}{\rho_{i}^{\prime}} \frac{\mathrm{d}}{\mathrm{d} r}\left[\frac{\rho_{i}}{g_{i}^{2}}\left(\rho_{i}^{\prime}\right)^{2}\right]=\chi \mu c^{2} \rho_{i}^{2}
$$

Assume (and further prove) that at the sphere center

$$
\rho_{i}(r=0)=0
$$

For the sphere with constant density $\mu$, the solution of Equation (37) that satisfies the regularity condition at the sphere center is

$$
g_{i}^{2}=\frac{\left(\rho_{i}^{\prime}\right)^{2}}{1-\left(\chi \mu c^{2} / 3\right) \rho_{i}^{2}}
$$

For $\rho(r)=r$ Equations (36) and (39) coincide with the Schwarzchild solutions in Equations (18) and (22). However, in contrast to Equations (18) and (22), the obtained equations include the derivative $\rho^{\prime}(r)$.

\subsection{Internal Space}

Now, we need to specify the function $\rho(r)$. Recall that the main shortcoming of the Schwarzchild solution is the discrepancy between the Euclidean form of the sphere mass which allows us to satisfy the boundary condition $g_{e}(R)=g_{i}(R)$ and the actual mass (30) which corresponds to the Riemannian internal space of the sphere.

Note that the mass in Equation (30) depends on gravitation. Being discussed by, e.g. Zeldovich and Novikov [14], the dependence of mass on gravitation, does not seem to correspond to the principle of equivalence of gravitational and inertial masses. Indeed, the inertial mass shows itself under the action of forces whose nature is different from the nature of gravitation. Anyway, as known [3], any assumption may exist in GRT if the resulting solution is feasible. Thus, suppose that gravitation does not affect the sphere mass which remains the same that in the absence of gravitation and corresponds to the Euclidean space. As follows from Equation (29), the sphere mass corresponds to the Euclidean space if

$$
g_{i} \rho_{i}^{2}=r^{2}
$$

which is the equation for the function $\rho_{i}(r)$. Now, since the mass is Euclidean, Equation (23) of the Schwarzchild solution is valid and we can transform Equation (39) to the following final form:

$$
g_{i}^{2}=\frac{\left(\rho_{i}^{\prime}\right)^{2}}{1-r_{g} \rho_{i}^{2} / R^{3}}
$$

Substituting Equation (41) in Equation (40), we arrive at the following differential equation for the function $\rho_{i}(r)$ :

$$
\rho_{i}^{\prime} \rho_{i}^{2}=r^{2} \sqrt{1-r_{g} \rho_{i}^{2} / R^{3}}
$$

The solution of this equation which satisfies the condition in Equation (38) is

$$
F_{i}\left(\rho_{i}\right)=\frac{2 r_{g} r^{3}}{3 R^{4}}
$$


where

$$
F_{i}\left(\rho_{i}\right)=\sqrt{\frac{R}{r_{g}}} \sin ^{-1}\left(\frac{\rho_{i}}{R} \sqrt{\frac{r_{g}}{R}}\right)-\frac{\rho_{i}}{R} \sqrt{1-\frac{r_{g} \rho_{i}^{2}}{R^{3}}}
$$

Equation (42) implicitly specifies the function $\rho_{i}(r)$ which changes from $\rho_{i}=0$ that corresponds to the sphere center to $\rho_{i}(R)=\rho_{R}$ that corresponds to the sphere surface. Taking $r=R$ in Equation (42), we arrive at the following implicit equation for $\rho_{R}$ :

$$
F_{i}\left(\rho_{R}\right)=\frac{2 r_{g}}{3 R}
$$

\subsection{External Space}

The metric coefficients for the external space must satisfy the asymptotic conditions in Equations (35) and the following boundary conditions on the sphere surface $r=R$ :

$$
g_{e}(R)=g_{i}(R), \rho_{e}(R)=\rho_{i}(R)
$$

To satisfy these conditions, assume that Equation (40) is valid not only for the internal space, but for the external space as well, i.e.,

$$
g_{e} \rho_{e}^{2}=r^{2}
$$

Matching Equations (40) and (45), we can conclude that if the second boundary condition in Equations (44) is satisfied, the first of these conditions is satisfied automatically because $r$ is continuous. Substituting the first equation in Equations (36) in Equation (45), we get the following differential equation for the function $\rho_{e}(r)$ :

$$
\rho_{e}^{\prime} \rho_{e}^{2}=r^{2} \sqrt{1-\frac{r_{g}}{\rho_{e}}}
$$

The solution of equation (46) which satisfies the second boundary condition in Equations (44) is

$$
F_{e}\left(\rho_{e}\right)-F_{e}\left(\rho_{R}\right)=\frac{1}{3}\left(\frac{r^{3}}{R^{3}}-1\right)
$$

where

$$
\begin{aligned}
F_{e}\left(\rho_{e}\right)= & \frac{1}{R^{3}}\left(\frac{1}{3} \rho_{e}^{2}+\frac{5}{12} r_{g} \rho_{e}+\frac{5}{8} r_{g}^{2}\right) \sqrt{\rho_{e}\left(\rho_{e}-r_{g}\right)} \\
& +\frac{5}{8}\left(\frac{r_{g}}{R}\right)^{3} \ln \left(\sqrt{\frac{\rho_{e}-r_{g}}{R}}+\sqrt{\frac{\rho_{e}}{R}}\right)
\end{aligned}
$$

This solution is valid for $\rho_{R} \leq \rho_{e}<\infty$ where $\rho_{R}$ corresponds to the sphere surface. For the external space, we need to check the asymptotic conditions in Equations (35). Dividing Equation (47) by $\rho_{e}^{3}$ and taking $\rho_{e} \rightarrow \infty$, we can readily prove that $\rho_{e} \rightarrow r$. Differentiating Equation (47) with respect to $\rho_{e}$ and dividing the result by $\rho_{e}^{3}$, we can prove that the second condition in Equations (35) is 
also valid. Thus, for $\rho_{e} \rightarrow \infty$, Equations (36) reduce to the Schwarzchild solutions in Equations (18) which, in turn, degenerate into the CGT solutions in Equations (17).

\subsection{Critical Radius}

Consider Equation (47) from which it follows that the real solution exists if $\rho_{e} \geq$ $\rho_{R} \geq r_{g}$. Thus, the minimum possible value of the function $\rho_{R}$ that corresponds to the sphere surface is $r_{g}$ specified by Equation (2). Taking $\rho_{R}=r_{g}$ in Equation (43), we arrive at the following equation for the minimum possible value of the sphere radius $R_{g}$ :

$$
\sqrt{\frac{R_{g}}{r_{g}}} \sin ^{-1}\left(\frac{r_{g}}{R_{g}} \sqrt{\frac{r_{g}}{R_{g}}}\right)-\frac{r_{g}}{R_{g}} \sqrt{1-\left(\frac{r_{g}}{R_{g}}\right)^{3}}=\frac{2 r_{g}}{3 R_{g}}
$$

The solution of equation (48) is $R_{g}=1.115 r_{g}$. For the sphere with $R<R_{g}, \rho_{R}<$ $r_{g}$ and the solution in Equation (48) becomes imaginary.

\subsection{Comparison with the Schwarzchild Solution}

As follows from the foregoing derivation, the obtained solution gives the critical radius similar to the horizon of events radius of the Black Hole in the Schwarzchild solution. However first, this radius does not coincide with $r_{g}$ which actually follows from CGT. Second, for the sphere with the critical radius $R_{g}$, the solution is not singular. Indeed, Equations (40), (45) and (47) for $r=R_{g}$ yield $g_{e}(R)=$ $g_{i}(R)=1.243$ and $\rho_{e}(R)=\rho_{i}(R)=0.8968 \mathrm{R}$. Third, for sphere with radius $R<R_{g}$, the solution becomes imaginary which means (in accordance with A. Einstein prediction) that GRT equations are not valid in this case. The dependences $\rho(r)$ and $g(r)$ corresponding to the limiting case $R=R_{g}=1.115 r_{g}$ are shown in Figure 1 with solid lines. Dashed lines correspond to the limiting case $\left(R=r_{g}\right)$ of the Schwarzchild solution.

As can be seen, the obtained solution coincides with the Schwarzchild solution at $r=0$ and $r \rightarrow \infty$. The most pronounced difference is observed in the vicinity of the sphere surface. For $R=r_{g}$, the Schwarzchild solution is singular at $r=R$, whereas the obtained solution is finite.

To proceed, find the pressure $p$ in the fluid. Taking $\sigma_{r}=-p$ in Equation (12) and substituting $g_{i}$ from Equation (41), we arrive at the following equation for $h_{i}$ :

$$
\frac{h_{i}^{\prime}}{h_{i}}=\frac{1}{2} \rho_{i} \rho_{i}^{\prime}\left[\frac{1}{1-r_{g} \rho_{i}^{2} / R^{3}}\left(\frac{1}{\rho_{i}^{2}}+\chi p\right)-\frac{1}{\rho_{i}^{2}}\right]
$$

in which, as earlier, $(\ldots)^{\prime}=\mathrm{d}(\ldots) / \mathrm{d} r$.

Changing $r$ to a new variable $\rho_{p}$ we can transform Equation (49) to

$$
\frac{1}{h_{i}} \frac{\mathrm{d} h_{i}}{\mathrm{~d} \rho_{i}}=\frac{1}{2} \rho_{i}\left[\frac{1}{1-r_{g} \rho_{i}^{2} / R^{3}}\left(\frac{1}{\rho_{i}^{2}}+\chi p\right)-\frac{1}{\rho_{i}^{2}}\right]
$$

Integration of Equation (50) allows us to satisfy the boundary condition $h_{e}(R)$ $=h_{i}(R)$. The pressure $p$ entering Equation (50) is specified by Equation (15). 


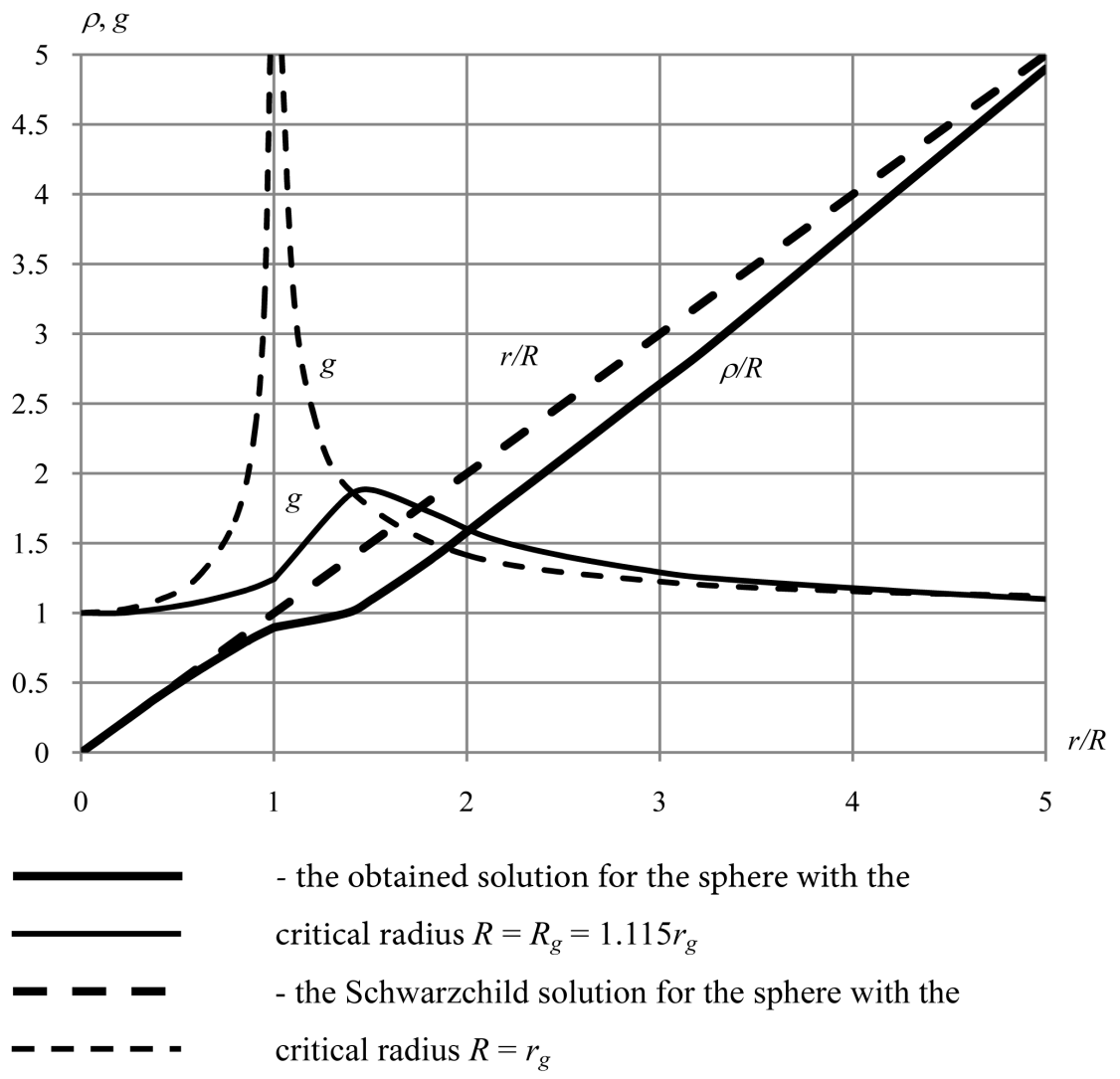

Figure 1. Dependences of the space metric coefficients on the radial coordinate.

Changing $r$ to $\rho_{i}$ in this equation, we get

$$
\frac{\mathrm{d} p}{\mathrm{~d} \rho_{i}}+\frac{1}{h_{i}} \frac{\mathrm{d} h_{i}}{\mathrm{~d} \rho_{i}}\left(p+\mu c^{2}\right)=0
$$

Substituting Equation (50) in Equation (51), we obtain the following equation for the pressure:

$$
\frac{\mathrm{d} p}{\mathrm{~d} \rho_{i}}+\frac{r_{g} \rho_{i}}{2\left(R^{3}-r_{g} \rho_{i}^{2}\right)}\left(p+\mu c^{2}\right)\left(1+\frac{3 p}{\mu c^{2}}\right)=0
$$

As can be seen, Equation (52) is analogous to Equation (26) and its solution which satisfies the boundary condition $p\left(\rho_{R}\right)=0$ is similar to Equation (28), i.e.,

$$
p=-\mu c^{2} \frac{\sqrt{1-r_{g} \rho_{i}^{2} / R^{3}}-\sqrt{1-r_{g} \rho_{R}^{2} / R^{3}}}{\sqrt{1-r_{g} \rho_{i}^{2} / R^{3}}-3 \sqrt{1-r_{g} \rho_{R}^{2} / R^{3}}}
$$

The pressure at the sphere center $\left(\rho_{i}=0\right)$ is

$$
p_{0}=\mu c^{2} \frac{1-\sqrt{1-r_{g} \rho_{R}^{2} / R^{3}}}{3 \sqrt{1-r_{g} \rho_{R}^{2} / R^{3}}-1}
$$

The denominator of Equation (54) becomes zero if $R=1.013 r_{g}$ which is less than the critical radius $R_{g}=1.115 r_{g}$. Thus, in contrast to the Schwarzcnild solution in Equation (31), the pressure is not singular. For the sphere with the critical radius $R=R_{g}$, we get $p_{0}=0.808 \mu c^{2}$. The dependences of the normalized 
pressure on the radial coordinate are presented in Figure 2. Solid line demonstrates the obtained solution, Equation (53), for the sphere with the critical radius $R=R_{g}$. Dashed line corresponds to the Schwarzchild solution, Equation (28), for the sphere with the limiting radius $R=R_{s}=9 / 8 r_{g}$.

Thus, the critical radius is $R_{g}=1.115 r_{g}$. Using Equation (2) for $r_{g}$ we get

$$
R_{g}=2.23 \frac{m G}{c^{2}}
$$

As shown by Vasiliev and Fedorov [15], for the sphere with radius $R_{g}$ (as for the Black Hole) the escape velocity is equal to velocity of light and, following $P$. Laplace, this sphere can be referred to as the Dark Star. Naturally, the parameters of this object are different from those found by Laplace.

Thus, we can conclude that GRT equations are valid for the problem under study if $R \geq R_{g}$. The sphere with $R<R_{g}$ is statically stable. Assume that due to high gravitation the Dark Star with radius $R \leq R_{g}$ attracts some additional fluid mass with the same density and the star mass becomes higher. Then, in accordance with Equation (55) the critical radius increases in proportion to the mass. However, the sphere radius $R$ is proportional to $m^{1 / 3}$. So, the inequality $R<R_{g}$ remains valid and the Dark Star remains invisible.

The obtained result is of a qualitative nature because it corresponds to the fluid sphere with constant density, i.e., to the object which is not quite realistic.

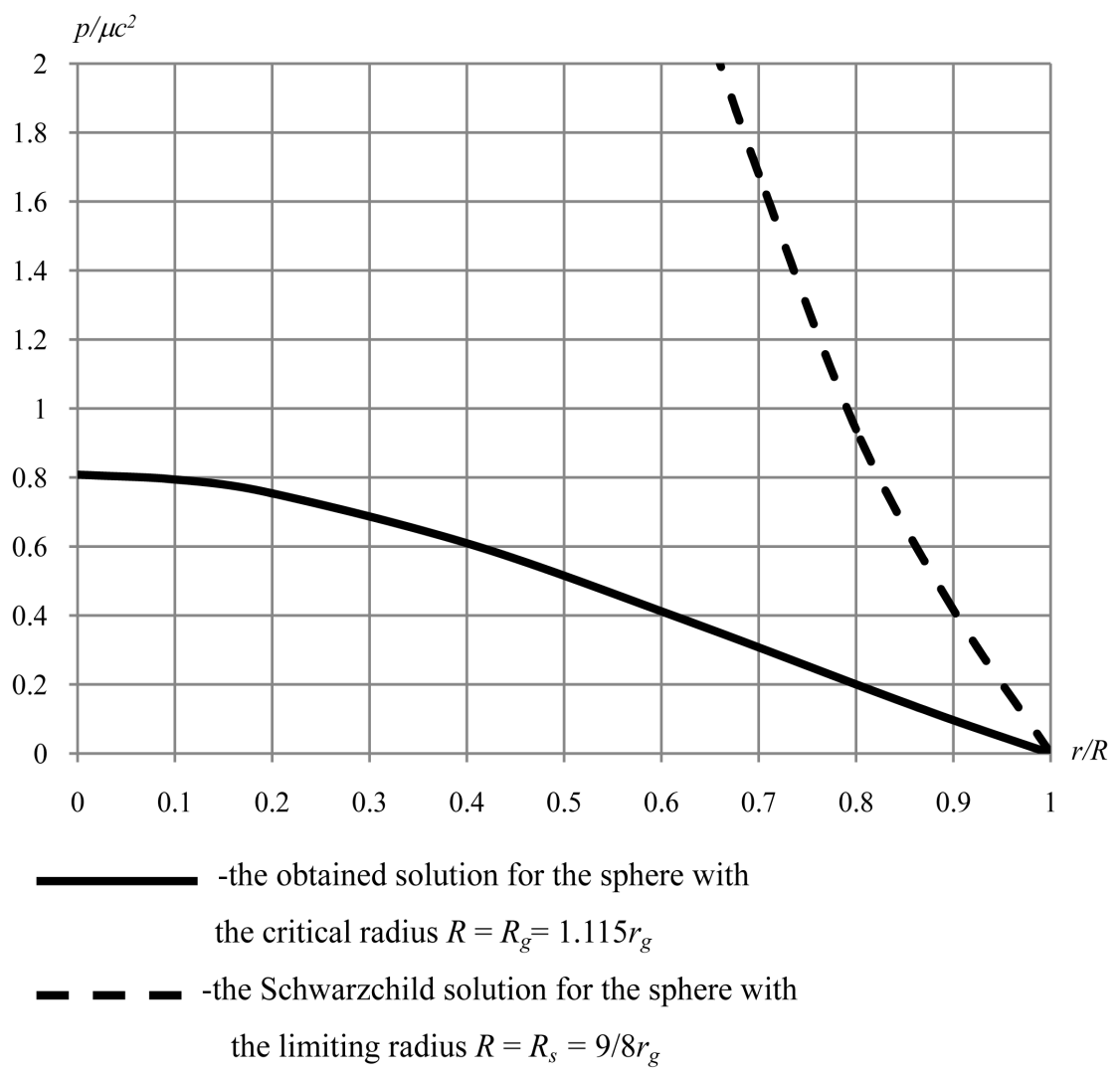

Figure 2. Dependences of the normalized pressure in the fluid on the radial coordinate. 


\section{Conclusion}

As follows from the foregoing discussion, the singular Schwarzchild solution of the spherically symmetric GRT problem satisfies the boundary condition for the space component of the metric tensor on the sphere surface only if the sphere mass is Euclidean which is not the case in GRT.

The proposed solution of the problem based on the assumption according to which the gravitation does not affect the sphere mass, in contrast to the Schwarzchild solution, is not singular and specifies the critical radius of the sphere limiting the application of GRT equations to the spheres whose radii are equal or larger than the critical value. The obtained solution is valid for the sphere consisting of a perfect incompressible fluid. Thus, the objects that are similar to Dark Stars in the Classical Gravitation Theory can follow from the General Relativity Theory.

\section{Acknowledgements}

The author thanks L.V. Fedorov for collaboration.

\section{References}

[1] Thorn, K.S. (1994) Black Holes and Time Warps-Einstein's Outrages Legacy. W.W. Norton and Co., New York, London.

[2] Novikov, I.D. (1985) Black Holes and Universe. Molodaya Gvardia, Moscow. (In Russian)

[3] Synge, J.L. (1960) Relativity: The General Theory. North Holland, Amsterdam.

[4] Vasiliev, V.V. and Fedorov, L.V. (2015) Applied Physics Research, 7, 10-17. https://doi.org/10.5539/apr.v7n3p10

[5] Vasiliev, V.V. and Fedorov, L.V. (2014) Mechanics of Solids, 4, 15-29.

[6] Feinman, R.F., Morinigo, F.B. and Wagner, W.G. (1959) Feinman Lectures on Gravitation. Addison-Wesley Publishing Company, Boston.

[7] Landau, L.D. and Lifshitz, E.M. (1988) Field Theory. Nauka, Moscow. (In Russian)

[8] Fock V. (1959) The Theory of Space, Time and Gravitation. Pergamon Press, London.

[9] Logunov, A.A. (2006) Relativistic Theory of Gravitation. Nauka, Moscow. (In Russian).

[10] Einstein, A. (1939) Annals of Mathematics, 40, 922-936. https://doi.org/10.2307/1968902

[11] Vasiliev, V.V. (2010) Mechanics of Solids, 45, 205-213. https://doi.org/10.3103/s0025654410020068

[12] Vasiliev, V.V. and Fedorov, L.V. (2014) Applied Physics Research, 6, 40-49. https://doi.org/10.5539/apr.v6n3p40

[13] Weinberg, S. (1973) Gravitation and Cosmology. North Holland, Amsterdam.

[14] Zeldovich, Ya.B. and Novikov, I.D. (1971) Theory of Gravitation and Evolution of Stars. Nauka, Moscow. (In Russian)

[15] Vasiliev, V.V. and Fedorov, L.A. (2015) Applied Physics Research, 7, 84-92. https://doi.org/10.5539/apr.v7n3p84 
Submit or recommend next manuscript to SCIRP and we will provide best service for you:

Accepting pre-submission inquiries through Email, Facebook, LinkedIn, Twitter, etc. A wide selection of journals (inclusive of 9 subjects, more than 200 journals)

Providing 24-hour high-quality service

User-friendly online submission system

Fair and swift peer-review system

Efficient typesetting and proofreading procedure

Display of the result of downloads and visits, as well as the number of cited articles Maximum dissemination of your research work

Submit your manuscript at: http://papersubmission.scirp.org/

Or contact jmp@scirp.org 